\title{
Social Media Usage and Shopping Preferences: an Empirical Investigation
}

\author{
Joseph Vithayathil \\ Washington State University \\ joseph.vithayathil@wsu.edu
}

\author{
Majid Dadgar \\ University of San Francisco \\ mdadgar@usfca.edu
}

\author{
John Kalu Osiri \\ University of Nebraska-Lincoln \\ jkosiri@unl.edu
}

\begin{abstract}
We empirically explore the associations between social media usage at home and shopping preferences using survey data. We focus on popular retail firms including brick-and-mortar firms such as Walmart, Target, Nordstrom, and Best Buy, and online retailers, such as Amazon, Walmart, Target, and Best Buy. Social media usage of popular platforms such as Facebook, Twitter, LinkedIn, and Skype are analyzed. We draw on Media Richness Theory (MRT) and Strength of Weak Ties from Social Network Analysis (SNA) and related theories to explain our results. Our results have important implications for social marketing campaigns and social media policies for consumer retail firms.
\end{abstract}

\section{Introduction}

Today, with the rapid growth of social media, most organizations seek ways to use social media [20] for profits and performance. Over half of social media users are engaged with at least one retailer's social media platform such Facebook, LinkedIn, and Twitter. Well known and popular retailers actively strategize business models to take advantage of such an opportunity [30]. Retail stores who are developing e-commerce platforms rely on web 2 technologies such as popular social media platforms and social networks to increase revenue and improve business models. McKinsey [3] reports that marketers use popular social media platforms such as Facebook and Twitter to create buzz around their products to increase sales and gain larger returns from investments. For example, Nordstrom now expects online sales to make up 30 percent of its business by 2020, up from 8 percent in $2010^{1}$. Nordstrom customers who shop both online and in stores spend three to four times as much at Nordstrom than those who shop just using one channel ${ }^{1}$.

Social media are changing the way organizations function and evolve on many fronts. Social media are transforming businesses and their relationships with

\footnotetext{
${ }^{1}$ http://www.bloomberg.com/gadfly/articles/2016-03-09/nordstroms-profits-zapped-by-online-sales
}

society, customers, and the marketplace [1]. Social media are transforming marketing approaches through which businesses understand consumer behavior and consumer preferences, to predict their demands [16,32].

Social media platforms allow a community of consumers to discuss and share ideas about certain brands, products or services [5]. Social media can enable companies to develop new methods and techniques to communicate with customers and suppliers [5]. Unlike traditional media, managers can use social media to design marketing messages and communicate them with millions of consumers at the same time [27]. Furthermore, firms use social media to engage customers to attain organizational objectives [28]. Enterprise social media which are enterprisespecific forms of social media help managers to know their customers better, and make informed decisions [17]. Many firms use social media to respond to the customer and retailer criticism about their brand and about products [33]. Consumer attitude and preferences reflected in product reviews in social media can influence firm value [26,34]. Social media can be very useful tool to help firms to maintain a positive image and survive turbulence in the market $[22,24]$.

Firms spend millions of dollars in social media marketing, but may not fully realize the benefits that can be derived from the social media platform because the firm is not managing social media purposefully and systematically [15]. Very few studies have investigated the impact of different kinds of popular social media on shopping preferences from popular brick-and-mortar stores, and online retail stores. Our study is designed to build this area of research for scholarly and practitioner value, and is conducted at the intersection of social media, social networks, and consumer behavior. (Figure $1)$.

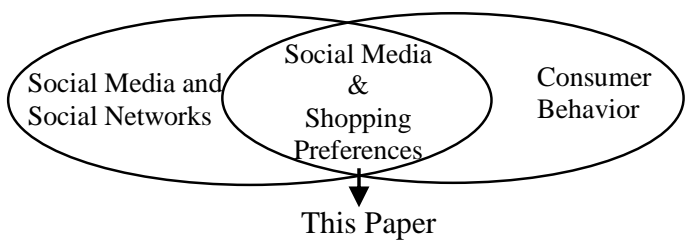

Figure 1. Research Boundaries. 
The primary focus of this study is to investigate the nature of the associations between consumer shopping preferences, and various popular and well known social media platforms such as Facebook, Twitter, Skype, LinkedIn, and Other Social Media. Our study considers brick-and-mortar stores as well as online stores.

We investigate the associations between popular social media platforms and retail shopping stores to address the following research questions: (i) how is the use of popular social media at home associated with shopping preferences with respect to specific retail stores? (ii) what theories and frameworks can best explain the empirically observed associations? (iii) what are the implications of these results so that firms can benefit from the use of social media? and (iv) what may best explain any lack of significant associations?

Our findings show that using Facebook at home is positively associated with shopping from Target's brick-and-mortar stores, use of LinkedIn at home is positively associated with shopping from brick-andmortar Nordstrom stores, Skype usage at home is positively associated with shopping from brick-andmortar Walmart stores, and use of Other Social Media is significantly associated with shopping from Target and Nordstrom. Other Social Media is a catchall designation to capture public social media platforms that are not specified (such as Pinterest) and also includes retailer-specific, consumer-specific and firm-specific social media platforms.

Our findings show significant associations with online markets. Use of Facebook and Skype at home and Amazon, use of Twitter at home and BestBuy, use of Other Social Media and Walmart are positively associated.

The rest of the paper is organized as follows. First we discuss the theoretical background, review the literature, and propose our hypotheses. Next, we discuss our data and our model, and explain our results and robustness checks. We discuss our results and their implications followed by the conclusion.

\section{Theoretical background, literature review, and hypotheses}

In this section we describe the theoretical background for our study followed by a review the relevant literature and development of our hypotheses.

\subsection{Theoretical background}

Media richness theory (MRT) [6] and theory of the strength of weak ties $[13,14]$ have been widely used to explain the effects and roles of social media in organizations [7]. In this section we provide an overview, and describe the two theories and their fit for our research.

MRT is used to explain the impact of computermediated communications [19], effects of video communication on decision making [8], and people's choice of electronic mail versus voice mail [10]. MRT explains media use be examining the fit between the complexity of the task information and media richness. Richer media offers higher communicative capabilities, and improves task performance [6]. More complex information is better communicated with richer media [7]. For example, information characterized by high level of complexity and various possible interpretations is better communicated through the medium of face-toface meeting in which two parties can clarify ambiguities arising from the information. Hence, they can perform the task based on clear information. Media with lower levels of richness such as computer-based social media, are better suited to communicate information that is lower in complexity [9].

Kaplan and Haenlein [20] provides a framework for assessing media richness. Their framework shows how media can differ based on the richness attribute. We have adapted their framework to describe the social media we study based on their richness attribute as shown in Figure 2. Based on this framework, Twitter as a microblogging medium has the lowest level of richness, the social networking platforms of Facebook and LinkedIn have medium richness. Skype which is a text, audio and video communication social medium has the highest richness in our set of social media platforms.

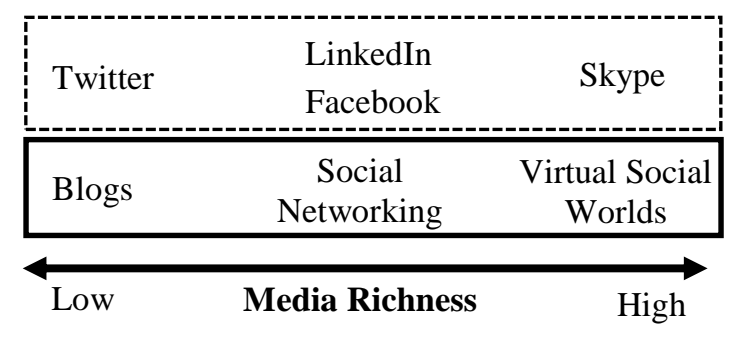

Figure 2. Social Media overlay on Media Richness adapted from Kaplan and Haenlein [20]

In order to understand and explain the use of social media by individuals and understand the corresponding organizational benefits we draw on the theory of strength of weak ties (SWT) [13]. SWT shows that weak ties provide value to those individuals who are connected with weak ties (such as casual acquaintances) because these weak ties provide access to informational resources and opportunities that strong ties (close friends) do not have [14]. SWT has been used to analyze 
and explain the role of word-of-mouth referral behavior [2], social support networks [4], social media design [11], occupational status attainment based on social resources [25], information diffusion in online social networks [36], informal communication and its impact on collaborative work [35], social value of online interactions [12], and networked communities [21].

Zhang and Rosson [35] argue that individuals with weak ties easily and quickly establish mutual trust. Once mutual trust is established it is likely that individuals share important and valuable information. They explore the effects of using Twitter on informal communication at work. Their findings capture set of qualities that explain why people use a micro-blogging platform like Twitter. For example, the brevity and real-time qualities of the posts on Twitter, among others, makes Twitter attractive to the users.

Gilbert and Karahalios [11] investigate tie strength and its relevance to social media. Their findings provide guidelines for designing social media. They suggest several theoretical dimensions for the strength of tie, such as interaction frequency and communication reciprocity. They argue that understanding the strength of ties can help design social media, for example, with better privacy control. For example, social media users may have different privacy concerns for the weak ties than the strong ties. Recognizing the difference between the strength of ties will guide design of the privacy levels in social media.

Grabowicz et al. [12] analyze the social features of Twitter. Their analysis shows that Twitter is organized by certain groups that form its active environment. They introduce the role of intermediary ties in the transmission of the new event information. Their findings show that the weak ties between groups transfer new information. Intermediary ties that belong to different groups work as information brokers and disseminate new information to individuals in different groups.

Kavanaugh et al. [21] show that weak ties who use communication media can better educate communities and organize collective actions. In the communities leaders and those who are members of more than one group are considered weak ties. Their results show that weak ties who use internet-based communication in the networked communities are socially active and engaged in the community meetings compared to others who are not weak ties across different groups. Their findings show that internet can improve information diffusion and social activities.

\subsection{Literature review and hypotheses}

There are two broad streams of research in social media usage and consumer behavior. These streams of research are business value $[5,17,26,34]$ and communication $[24,27,28,33]$. We provide a review of the literature and describe these two streams of research.

Several studies use econometric models to analyze the relationships between the consumer preferences and marketing efforts in the e-commerce marketplace. For example, Luo et al. [26] use econometric models to examine and explain the relationships between usergenerated reviews in social media and the firm value. Jiang et al. [17] suggest that managers can use discussion content contained in firm-specific social media to understand the concerns of stakeholders to make informed decisions. Their results demonstrate the manner in which social media can be used to better understand stakeholder reactions to the major events in a firm.

Xia [33] examines the impact of a firm's responses to consumer criticism through social media. He draws on psychology and social relationship literature to define vulnerability and the positive effect of vulnerability on building effective relationships with customers via social media. His results show that a more vulnerable response generates better positive perceptions among consumers compared to a defensive response. Expectation-disconfirmation theory is used to explain how consumers evaluate responses posted in social media regarding consumer products.

Mangold and Faulds [27] examine non-traditional means of communication enabled by social media between consumers. They compare traditional and nontraditional communications enabled by social media based on integrated marketing communications (IMC) which is used to develop communication strategies to achieve organizational and strategic objectives. They propose a new communication paradigm to highlight the important role of the interactions and communications between consumers in the marketplace, on social media platforms.

Kuvykaite and Piligrimiene [24] note that social media enables many-to-many communication platforms for firms. They draw on identity theories to investigate the relationship between communication enabled by social media and its impact on firm image. Their results show that a quick communication from the company in the social media will form a positive image. Martini et al. [28] investigate how social media can help firms to communicate with customers and to engage them with the firm's activities. They draw on Pickering's "mangle" theory and Jones' subsequent metaphor of "double dance of agency" to investigate the mutual relationship between the human and technology agencies within social media platforms. Their results illustrate the manner in which humans and technology co-evolve over time and influence each other. 
Today, major retail stores have a digital presence on social media platforms. As of March 24, 2016, Walmart, for example, has more than 32 million likes and 13 million page visits on its Facebook page. Retail stores such as Target, Best Buy, and Nordstrom have the same significant presence within the social media space. Some reports show that over $40 \%$ of people have "friended" a brand on Facebook and $20 \%$ of tweets are about products such as invitations for product information, answers or responses from peers, or direct communication by brand representatives ${ }^{2}$. $56 \%$ of Twitter users say they use Twitter's microblogging and communication service for business purposes ${ }^{2}$. Best Buy has over 2,500 employees helping customers via Twitter as part of their @ Twelpforce initiative ${ }^{2}$.

Prior literature shows that social media can be an effective marketing tool in the retail market [15,18,23,29,30,31]. Kim and Ko [23] argue that social media make it easier and less costly for retailers to perform marketing activities. They show that retailers and luxury brands that are present on social media can significantly improve their reputation and image. They report that $70 \%$ of the consumers have used social media to collect information about retailers, and about $50 \%$ of the consumers have made a purchase based on the information they have found on social media. There has been $80 \%$ increase in social media expenditures in the retail industry in the US [15].

Retailers use social media to engage their customers. Sashi [31] shows that retailers can use social media to enhance their relationships with their loyal customers and fans. His analysis shows that customer engagement is established through a cycle of steps. Retailers should first reach out, connect, and interact with their customers. Next they should address their needs and questions. And lastly, they should retain their loyalty and commitment over time. Higher customer engagement will help retailers and popular brands to transform regular customers into loyal customers [31].

"Likes" regarding a social media post or message on a social media platform [18] has important consumer effects. Jones et al. [18] show that making connections with users on social media can translate into positive brand image and purchase intentions. They argue that revealing the demographics of the users who have "Liked" a post, for example, on Facebook, can affect the brand image in other users' minds. Rapp et al. [30] recognize a contagious effect in social media usage that could help retailers to enhance certain performance measures such as consumer-retailer loyalty and brand performance.

Therefore we hypothesize that:

\footnotetext{
2 http://pamorama.net/2010/02/22/31-social-media-stats-andanecdotes/
}

H1: Using social media at home is positively associated with shopping at Walmart, Target, Best Buy, and Nordstrom brick-and-mortar retail stores.

H2: Using social media at home is positively associated with shopping from Amazon, Best Buy, Walmart, and Target online stores.

Our research model is shown in Figure 3.

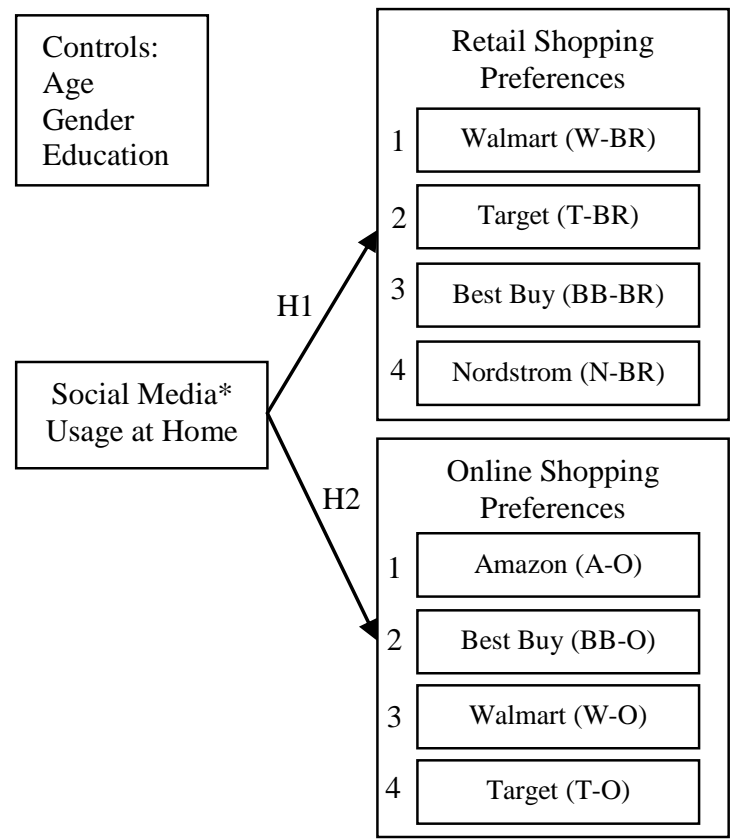

Figure 3. Research model

* Social media tested in our study: Facebook (FB), Twitter, LinkedIn, Skype, and Other Social Media.

\section{Data and model}

A total of 274 subjects $(\mathrm{N}=274)$ participated in this study by responding to survey questions. The participants were working students of a public university in the state of Washington. Sample research questions are provided in Appendix A and B. The summary demographics of the respondents are provided in Table 1.

A logistic regression was conducted to predict the relationship between social media usage at home and at work, with shopping preferences from brick-and-mortar stores and online stores. The model specification is as follows: 
Shopping_Preferences $s_{i}=\hat{\beta}+\beta_{1 i} . F B_{-} H_{i}+$ $\beta_{2 i}$. Twitter_ $H_{i}+\beta_{3 i}$. LinkedIn_ $H_{i}+$ $\beta_{4 i}$. Skype $H_{i}+\beta_{5 i}$. OtherSM_ $H_{i}+$ Controls $+\varepsilon_{i}$

Table 1. Demographics of the subjects

\begin{tabular}{|l|l|c|}
\hline Variable & $20-35$ years old & Percentage \\
\hline \multirow{4}{*}{ Age } & $35-50$ years old & 72 \\
\cline { 2 - 3 } & $50-65$ years old & 23 \\
\hline \multirow{4}{*}{ Gender } & Female & 5 \\
\cline { 2 - 3 } & Male & 58 \\
\hline \multirow{4}{*}{ Education } & High school & 42 \\
\cline { 2 - 3 } & Community college & 41 \\
\cline { 2 - 3 } & Four year college & 45 \\
\cline { 2 - 3 } & Graduate degree & 6 \\
\hline
\end{tabular}

In the next section we report the results from the analysis of our regression model from this surveybased empirical study.

\section{Results}

We examined the relationship between use of social media at home and shopping preferences from brickand-mortar and online retail stores. Specifically we have tested for the associations between the usage of the popular social media platforms: Facebook, Twitter, Skype, LinkedIn, and Other Social Media; and brickand-mortar retail stores: Walmart, Target, Best Buy, Nordstrom; and online retail stores: Amazon, Walmart, Target, and Best Buy.

Our results partially support our hypotheses as follows.

\subsection{H1: Using social media at home is positively associated with shopping at Walmart, Target, Best Buy, and Nordstrom brick-and-mortar stores.}

LinkedIn usage at home is positively associated, .505 (.170), with shopping from Nordstrom. This result supports our hypothesis. LinkedIn and is a social networking platform that is specifically designed for career and professional networking. Hence, our finding suggests that Nordstrom may be a preferred shopping destination for LinkedIn members who have career and professional interests. Nordstrom products may fit the needs of professionals who prefer to shop at upscale retailers because Nordstrom has a brand equity that is consistent with professional attire. The strength of weak ties of LinkedIn users enable digital word-of-mouth communications for the Nordstrom products which would encourage other professionals in the in the immediate and strong network to shop at brick-andmortar Nordstrom stores.

Using Skype at home is positively associated, .439 (.207), with shopping from Walmart physical store. This result supports our hypothesis. Skype based on media richness is considered a medium with high richness which can help weak ties to communicate and exchange information about products of Walmart. Walmart sells Skype prepaid cards which can encourage Walmart shoppers to more often use Skype services. Using a free rich highly rich medium such as Skype users can better communicate their ideas about the products. For example they can group chat, video chat, and show products at the same time. The fit between

Using Other Social Media is negatively associated with shopping from Target and positively associated with shopping from Nordstrom, respectively with -.322 (.128) and .419 (.167). The negative association between using Other Social Media and shopping from Target does not support our hypothesis. Other Social Media of consumer products vary in size and capabilities. Some social media are specifically designed to advertise deals and sales. The positive association for Nordstrom compared to the negative association for Target shows that deals and discounts of more expensive products, such as those by Nordstrom, are more attractive to the consumers. Weak ties tend to communicate about the discounts and deals of the luxury products by Nordstrom.

\subsection{H2: Using social media at home is positively associated with shopping at Amazon, Best Buy, Walmart, and Target online stores.}

We fins that the use of Facebook at home is positively associated, .450 (.199), with shopping online from Amazon. This result partially supports our hypothesis. Amazon's page on Facebook has more than 26 million likes. Amazon uses the benefits of this large user base to engage with its customers. For example users can post pictures of their products recently purchased from Amazon to communicate with customer service. Facebook as a social network site with a medium level of media richness based on MRT. Such media richness allows Amazon to show product recommendations based on users' "likes" on Facebook ${ }^{3}$. This merging between Facebook likes and Amazon recommendations makes purchasing products online from Amazon quicker and convenient. Therefore, such

\footnotetext{
${ }^{3}$ http://mashable.com/2010/07/27/amazon-facebookrecommendations/\#SbWPNE1SM5qQ
} 
convenient, rich and mutual conversation between Facebook users and Amazon can transform regular Facebook users to customers of Amazon.

Use of Twitter at home is positively associated, .475 (.199), with shopping online at Best Buy. This result supports our hypothesis. Twitter is used as a platform to build a following and a brand and it also engages customers ${ }^{4}$. The strength of weak ties plays an important role in Twitter to diffuse information between the members and groups [12]. Best Buy has partnered with Twitter to incorporate a 'Buy' button in the tweets so that users can directly purchase the products within the tweets $^{5}$. This capability can be very attractive to Twitter users and enables them to follow Best Buy on Twitter which leads consumers to purchase electronic products.

The use of Skype at home is negatively associated, .528 (.273), with shopping online from Amazon. This result does not support our hypothesis and is contrary to the hypothesis. This result suggests that those users who use Skype do not tend to shop at Amazon. A plausible explanation for this negative association is that Skype is high in media richness because of the inclusion of video calls. Hence, while Skype may be good for friends and family chat and video, it may not be a good fit for focused exchange of product information regarding Amazon products.

3.

Summary of the results are provided in Tables 2 and

Table 2. Summary of results for social media usage at home and shopping preferences from brick-and-mortar retail stores

\begin{tabular}{|l|l|l|l|l|l|}
\hline SM & DV & B & SE & Wald & p-value \\
\hline \multirow{4}{*}{ FB } & W-BR & .124 & .113 & 1.211 & .271 \\
\cline { 2 - 6 } & T-BR & .150 & .113 & 1.766 & .184 \\
\cline { 2 - 6 } & BB-BR & .108 & .137 & .621 & .431 \\
\cline { 2 - 6 } & N-BR & -.023 & .124 & .034 & .854 \\
\hline \multirow{4}{*}{ Twitter } & W-BR & .132 & .132 & .997 & .318 \\
\cline { 2 - 6 } & T-BR & .023 & .131 & .030 & .862 \\
\cline { 2 - 6 } & BB-BR & .204 & .155 & 1.735 & .188 \\
\cline { 2 - 6 } & N-BR & .134 & .136 & .965 & .326 \\
\hline \multirow{5}{*}{ LinkedIn } & W-BR & -.148 & .148 & 1.008 & .315 \\
\cline { 2 - 6 } & T-BR & .033 & .145 & .051 & .821 \\
\cline { 2 - 6 } & BB-BR & -.175 & .188 & .862 & .353 \\
\cline { 2 - 6 } & N-BR & .541 & .155 & 12.102 & $.001 * *$ \\
\hline \multirow{5}{*}{ Skype } & W-BR & .437 & .193 & 5.125 & $.024 * *$ \\
\cline { 2 - 6 } & T-BR & -.054 & .190 & .080 & .778 \\
\cline { 2 - 6 } & BB-BR & -.115 & .254 & .206 & .650 \\
\cline { 2 - 6 } & N-BR & -.187 & .212 & .785 & .376 \\
\hline \multirow{3}{*}{$\begin{array}{l}\text { Other } \\
\text { SM }\end{array}$} & W-BR & -.079 & .119 & .437 & .509 \\
\cline { 2 - 6 } & T-BR & -.287 & .119 & 5.761 & $.016 * *$ \\
\cline { 2 - 6 } & BB-BR & -.140 & .150 & .872 & .350 \\
\hline
\end{tabular}

${ }^{4}$ http://shiftingcareers.blogs.nytimes.com/2008/09/07/how-twittercan-help-at-work/?_r $=0$

\begin{tabular}{|l|l|l|l|l|l|}
\hline & N-BR & .352 & .130 & 7.369 & $.007 * *$ \\
\hline \multicolumn{6}{|c|}{$* p<.1 ; * * p<.05 ; * * * p<.001}$.
\end{tabular}

Table 3. Summary of results for social media usage at home and shopping preferences from online retail stores

\begin{tabular}{|c|c|c|c|c|c|}
\hline SM & DV & B & SE & Wald & p-value \\
\hline \multirow{4}{*}{ FB } & $\mathrm{A}-\mathrm{O}$ & .422 & .188 & 5.042 & $.025 * *$ \\
\hline & BB-O & -.011 & .185 & .003 & .954 \\
\hline & $\mathrm{W}-\mathrm{O}$ & -.122 & .193 & .400 & .527 \\
\hline & $\mathrm{T}-\mathrm{O}$ & -.016 & .159 & .010 & .921 \\
\hline \multirow{4}{*}{ Twitter } & $\mathrm{A}-\mathrm{O}$ & -.072 & .252 & .082 & .775 \\
\hline & BB-O & .496 & .191 & 6.747 & $.009 * *$ \\
\hline & $\mathrm{W}-\mathrm{O}$ & .327 & .246 & 1.758 & .185 \\
\hline & $\mathrm{T}-\mathrm{O}$ & -.082 & .196 & .176 & .675 \\
\hline \multirow{4}{*}{ LinkedIn } & $\mathrm{A}-\mathrm{O}$ & -.097 & .234 & .173 & .677 \\
\hline & BB-O & -.060 & .236 & .066 & .798 \\
\hline & $\mathrm{W}-\mathrm{O}$ & -.141 & .271 & .270 & .603 \\
\hline & $\mathrm{T}-\mathrm{O}$ & -.069 & .215 & .103 & .749 \\
\hline \multirow{4}{*}{ Skype } & $\mathrm{A}-\mathrm{O}$ & -.476 & .255 & 3.487 & $.062 *$ \\
\hline & BB-O & -.108 & .338 & .102 & .750 \\
\hline & $\mathrm{W}-\mathrm{O}$ & .323 & .299 & 1.166 & .280 \\
\hline & $\mathrm{T}-\mathrm{O}$ & -.212 & .309 & .474 & .491 \\
\hline \multirow{4}{*}{$\begin{array}{l}\text { Other } \\
\text { SM }\end{array}$} & $\mathrm{A}-\mathrm{O}$ & .204 & .213 & .919 & .338 \\
\hline & BB-O & -.222 & .211 & 1.110 & .292 \\
\hline & W-O & -.481 & .251 & 3.659 & .618 \\
\hline & $\mathrm{T}-\mathrm{O}$ & .074 & .168 & .197 & .658 \\
\hline
\end{tabular}

\section{Robustness check}

We added age, gender, and education as controls in the regression model to check for their effect on the results. None of the relationships significantly changed except for the usage of Twitter at home and shopping online from Walmart. The association between Twitter usage at home and shopping online from Walmart becomes significant at $\mathrm{p}<.1$, and the association between usage of Other Social Media and shopping online from Walmart becomes significant at $\mathrm{p}<.05$, when age, gender, and education are added as controls. For the Twitter usage and shopping online from Walmart, the p-value changes from .185 to .062 and its coefficient increases from .327 to .512 . For the association between usage of Other Social Media and shopping online from Walmart the p-value changes from .618 to .043 and its coefficient changes from -.481 to -.533 . These significant associations show the impact of education on the association between shopping from Walmart and social media usage. These results suggest that social media users with undergraduate degree who use Twitter tend to shop more from brick-and-mortar

5 http://www.bizjournals.com/pacific/blog/morning_call/2015/10/bestbuy-twitter-buy-now-deal.html 
Walmart stores than online compared to other social media users with alternative education.

\subsection{Alternative specifications}

5.1.1. Social media usage at home and at work for shopping from brick-and-mortar stores. We tested an alternative logistic regression model in which we included social media usage at work as a predictor. In the alternative model for the use of social media at home and social media use at work, and shopping from brickand-mortar stores, the following changes take place in our results.

The use of Facebook at home becomes significant at $\mathrm{p}<.1$ for shopping from brick-and-mortar Walmart stores. Its p-value changes from .271 to .078 , and its coefficient changes from .124 to .254 . This result shows that consumers may be using Facebook at work to follow on their favorite consumer products. The work environment may enable the strength of weak ties to facilitate exchange of shopping information via Facebook with colleagues at work. Working consumers may forego working responsibilities to occasionally check on information posted on Facebook about Walmart products. Such activity may or may not affect productivity at work which we do not address here.

The use of Twitter at home becomes significant at $\mathrm{p}<.1$ for shopping from Target brick-and-mortar stores. Its p-value changes from .862 to .071 and its coefficient changes from .023 to .406 . Twitter is a microblogging platform that enables users to exchange short messages fast, and has broadcast features. Given the lack of available leisure time at work, Twitter may be an ideal social media platform that has less workplace impact when used for personal purposes. Therefore, the combined, use of Twitter at home and at work can create a positive association.

The use of Other Social Media at home becomes non-significant for shopping from brick-and-mortar Nordstrom stores. Brand-specific social media and apps can provide information that will not be given otherwise. Therefore, if consumers continue using Other Social brand-specific Media at home, they can access deals and sales of the Nordstrom luxury products.

The use of Twitter at home becomes significant at $\mathrm{p}<.05$ for shopping from brick-and-mortar Best Buy store. Its p-value changes from .188 to .005 , and its coefficient changes from .204 to .677. Best Buy despite its relatively few number of followers, of about 800,00 , has been successful in engaging with users to promote its brand and products. Best Buy uses Twitter to enhance customer service and the customer experience by

\footnotetext{
${ }^{6}$ https://contently.com/strategist/2012/06/04/best-buy-uses-twitterto-enhance-customer-service
}

quickly answering customers questions and concerns on Twitter $^{6}$. Twitter users can broadcast their questions on Twitter at home and also at work. Hence, including the use of Twitter at work in the model makes the association significant for Best Buy.

5.1.2. Social media usage at home and at work for shopping on online stores. We tested an alternative logistic regression model in which we included social media usage at work as a predictor. In this alternative model the following changes take place in our results.

The use of Twitter and Skype become significant for shopping online from Walmart at $\mathrm{p}<.05$ and $\mathrm{p}<.1$ respectively. For Twitter usage at home, the p-value changes from .185 to .041 and its coefficient changes from .327 to .744 . For Skype usage at home, the p-value changes from .280 to .073 and its coefficient changes from .323 to .586. Walmart has the most active users on Twitter in our list of retailers with more than $40 \%$ being active users $^{7}$.

The association between using Skype and shopping online from Walmart becomes positive and significant when we include Skype use at work. This result suggests that consumers use Skype at work to talk about products and that Walmart is likely to be a preferred online retailer for such consumers. Employees may be using Skype at work for job-related purposes and for personal reasons. As employees use Skype for job-related purposes they may also talk about personal topics such as shopping consumer products. Alternatively, employees may be using Skype at work for personal reasons particularly because of its high media richness. The mixed use of Skype at home and at work is reflected in the positive association between Skype and shopping online at Walmart.

The association between using Other Social Media and shopping online from Walmart becomes significant at $\mathrm{p}<.1$. The $\mathrm{p}$-value of this association changes from .618 to .058 and its coefficient changes from -.481 to .632. This significant association shows that those who use Other Social Media at home and at work do not shop online from Walmart. This result suggests that the strength of weak ties is not in force when using Other Social Media to share information at work regarding shopping interests.

The use of Facebook and Skype become nonsignificant for shopping online from Amazon. A reason for this non-significant result could arise from banning employees from using social media at work. Hence, they may be unable to follow their favorite brands and product interests at work on Facebook and Skype.

\section{Discussion}

\footnotetext{
${ }^{7}$ http://www.prnewsonline.com/water-cooler/2012/11/20/on-twitterwalmart-has-the-most-active-followers/
} 
Our work has several implications. First, our results show a positive association between using LinkedIn at home and shopping from Nordstrom. LinkedIn can be an effective advertising platform for Nordstrom to promote its products to those target users who may be predisposed to shop at Nordstrom. Professionals who are interested in careers and professional networking are potential customers for higher end brands like Nordstrom. Attire offerings at Nordstrom may be considered professional and suited to the work environment. LinkedIn is a social networking site specifically designed for job seekers and employers with medium richness [6] which can facilitate targeting professionals to promote relevant products. This result shows that such social networks create an emerging market for retailers and advertisers with product that is suited for a professional social network.

We find that when social media usage is extended to include social media usage at work, the association between social media usage at home and shopping preferences are changed. The associations between use of Facebook and shopping from Walmart and use of Twitter and shopping from Target become positive and significant when use at work is included in the model. These results show that different marketing strategies may need to be modified depending on the user environment. For example, if employees are allowed to use social media at work, this usage extension can benefit Walmart. Walmart can invest more in their Facebook company pages and advertisements. Target can focus more on its social marketing campaigns for the Twitter platform. Target and Walmart can devise new strategies for their user base on Facebook and Twitter.

In contrast, some of the associations between usage at home and shopping preferences become nonsignificant when the social media usage at work is included. For example, including social media usage at work renders the associations between shopping online from Amazon and using Facebook and Skype nonsignificant. These results suggest that the effect of marketing campaigns for Amazon products may be attenuated if the target customers use Facebook and Skype at work. Based on this result Amazon could devise modified marketing strategies for improved efficacy. For example, they could exclude the display of their advertisements to those IP addresses that are corporate and target individual consumers. Amazon may also need to devise alternate marketing campaigns for the workplace.

Use of Skype at home is negatively associated with shopping online at Amazon. One implication of this result based on MRT is the lack of a good fit between the capabilities of a rich medium such as Skype and the task of promoting online products such as those offered by Amazon. This result suggests that the online retailers such as Amazon may not benefit from rich media to increase sale revenue. Social media users post their questions and concerns on social media to derive answers and benefits quickly when interested 8in a purchase. Rich media may make the process more complex. Therefore Amazon could engage customers and enhance its positive brand image by using media that is lower in richness. Our results show that use of Facebook and shopping online at Amazon have a positive association which supports this argument as MRT suggests that Facebook is lower in richness compared to Skype. A second factor is that the networking abilities of Skype are much lower than Facebook.

The use of Twitter at home is positively associated with shopping online at Best Buy. The implication of this result is that brands and companies that rely on fast and real-time customer service can benefit from social media that is low in richness. Specifically, broadcasting micro-blogging platforms such as Twitter offer rapid communications, but low media richness. Electronic products offered by Best Buy may generate immediate follow-up questions from customers. These questions can be best addressed in broadcasting media such as Twitter where an answer (A tweet, reply, or mention) will be seen by millions of followers. Therefore this one-to-many relationships between brand and the customers can be used to satisfy customers; immediate needs and complaints to enhance the brand image.

Our work has a number of limitations. First, we do not specifically identify Other Social Media which is a catchall for platforms not specifically named in the study. Hence, our concept of Other Social Media captures a variety of social media that fall outside the popular social media such as Facebook, Twitter, LinkedIn, and Skype. Other Social Media measures in our study may also represent enterprise or firm-specific social media.

Second, we do not model the combined use of the social media at home and at work as one variable. Third, the data is collected through a self-reported survey which has its own limitations. Although our results have important implications, it does not delve into the oneon-one relationships between certain retail brands and social media.

\section{Conclusion}

We draw on MRT and theory of the strength of ties to investigate the associations between the usage of popular social media and popular retailers. Our results based on logistic regression model show that choosing an appropriate social medium with certain media 
richness qualities can be important for popular retail brands as they impact their marketing campaigns. For example using social networking sites, such as Facebook and LinkedIn can be effective to increase sales of the products for the brick-and-mortar retailers. Rich media such as Skype may produce negative results for online retailers. Popular retail brands can benefit from less rich media such as Twitter. Social media such as Twitter with micro-blogging and broadcasting features can be used best for customer service.

Future research can extend our study to investigate the associations between other types of social media, beyond the popular brands in this study. Various marketing campaigns could be investigated with objective measures in the future research. For example marketing campaigns can be investigated, at granular levels, to understand which marketing strategies are effective with which specific social media, to increase sales and enhance customer satisfaction. Lastly, the nature of social media usage can be further investigated. Social media scholars can analyze how the quality of social media usage such as the time of day and length of use affects outcomes.

\section{References}

[1] Aral, S., Dellarocas, C., and Godes, D. Social Media and Business Transformation: A Framework for Research. Information Systems Research 24, 1 (2013), 3-13.

[2] Brown, J.J. and Reingen, P.H. Social Ties and Wordof-Mouth Referral Behavior*. Journal of Consumer Research 14, 3 (1987), 350-362.

[3] Bughin, J. Getting a sharper picture of social media's influence | McKinsey \& Company.

http://www.mckinsey.com/business-functions/marketingand-sales/our-insights/getting-a-sharper-picture-of-socialmedias-influence.

[4] Crowell, L.F. Weak ties: a mechanism for helping women expand their social networks and increase their capital. The Social Science Journal 41, 1 (2004), 15-28.

[5] Culnan, M.J., McHugh, P.J., and Zubillaga, J.I. How Large U.S. Companies Can Use Twitter And Other Social Media To Gain Business Value. MIS Quarterly Executive 9, 4 (2010), 243-259.

[6] Daft, R.L. and Lengel, R.H. Organizational Information Requirements, Media Richness And Structural Design. Management Science 32, 5 (1986), 554-571.
[7] Dennis, A.R., Fuller, R.M., and Valacich, J.S. Media, Tasks, and Communication Processes: A Theory of Media Synchronicity. MIS Quarterly 32, 3 (2008), 575-600.

[8] Dennis, A.R. and Kinney, S.T. Testing Media Richness Theory in the New Media: The Effects of Cues, Feedback, and Task Equivocality. Information Systems Research 9, 3 (1998), 256-274.

[9] Dennis, A.R., Kinney, S.T., and Hung, Y.C. Gender Differences In The Effects Of Media Richness. Small Group Research 30, 4 (1999), 405-437.

[10] El-shinnawy, M. and Markus, M.L. The poverty of media richness theory: explaining people's choice of electronic mail vs. voice mail. International Journal of Human-Computer Studies 46, 4 (1997), 443-467.

[11] Gilbert, E. and Karahalios, K. Predicting Tie Strength With Social Media. CHI 2009, (2009).

[12] Grabowicz, P.A., Ramasco, J.J., Moro, E., Pujol, J.M., and Eguiluz, V.M. Social Features of Online Networks: The Strength of Intermediary Ties in Online Social Media. PLoS ONE 7, 1 (2012), e29358.

[13] Granovetter, M.S. The strength of weak ties. American Journal of Sociology 78, 6 (1973), 1360-1380.

[14] Granovetter, M.S. The Strength of Weak Ties: A Network Theory Revisited. Sociological Theory 1, (1983), 201-233.

[15] Hanna, R., Rohm, A., and Crittenden, V.L. We're all connected: The power of the social media ecosystem. SPECIAL ISSUE: SOCIAL MEDIA 54, 3 (2011), 265273.

[16] Hill, S., Provost, F., and Volinsky, C. NetworkBased Marketing: Identifying Likely Adopters via Consumer Networks. Statistical Science 21, 2 (2006), 256-276.

[17] Jiang, S., Chen, H., Nunamaker, J.F., and Zimbra, D. Analyzing firm-specific social media and market: A stakeholder-based event analysis framework. Decision Support Systems 67, 0 (2014), 30-39.

[18] Jones, P., Comfort, D., and Hillier, D. What's in store? Retail marketing and corporate social responsibility. Marketing Intelligence \& Planning 25, 1 (2007), 17-30.

[19] Kahai, S.S. and Cooper, R.B. Exploring the Core Concepts of Media Richness Theory: The Impact of Cue Multiplicity and Feedback Immediacy on Decision 
Quality. Journal of Management Information Systems 20, 1 (2003), 263-299.

[20] Kaplan, A.M. and Haenlein, M. Users of the world, unite! The challenges and opportunities of Social Media. Business Horizons 53, (2010), 59-68.

[21] Kavanaugh, A., Reese, D., Carroll, J., and Rosson, M. Weak Ties in Networked Communities. Information Society 21, 2 (2005), 119-131.

[22] Kietzmann, J.H., Hermkens, K., McCarthy, I.P., and Silvestre, B.S. Social media? Get serious! Understanding the functional building blocks of social media. Special issue: social media 54, 3 (2011), 241-251.

[23] Kim, A.J. and Ko, E. Do social media marketing activities enhance customer equity? An empirical study of luxury fashion brand. Fashion Marketing and Consumption of Luxury Brands 65, 10 (2012), 14801486.

[24] Kuvykaite, R. and Piligrimiene, Z. Communication in Social Media for Company's Image Formation. Economics \& Management 18, 2 (2013), 305-317.

[25] Lin, N., Ensel, W.M., and Vaughn, J.C. Social Resources and Strength of Ties: Structural Factors in Occupational Status Attainment. American Sociological Review 46, 4 (1981), 393-405.

[26] Luo, X., Zhang, J., and Duan, W. Social Media and Firm Equity Value. Information Systems Research 24, 1 (2013), 146-163.

[27] Mangold, W.G. and Faulds, D.J. Social media: The new hybrid element of the promotion mix. Business Horizons 52, (2009), 357-365.

[28] Martini, A., Massa, S., and Testa, S. The firm, the platform and the customer: A "double mangle"

\section{Appendix A - Examples of social media usage questions}

Q1. Rate your weekly use of Facebook at Home

1. Very Low or None

2. Low

3. Moderate

4. High

5. Very High or Always

Q2. Rate your weekly use of Twitter at Work

1. Very Low or None

2. Low interpretation of social media for innovation. Information and Organization 23, 3 (2013), 198-213.

[29] Naylor, R.W., Lamberton, C.P., and West, P.M. Beyond the "Like" Button: The Impact of Mere Virtual Presence on Brand Evaluations and Purchase Intentions in Social Media Settings. Journal of Marketing 76, 6 (2012), 105-120.

[30] Rapp, A., Beitelspacher, L., Grewal, D., and Hughes, D. Understanding social media effects across seller, retailer, and consumer interactions. Journal of the Academy of Marketing Science 41, 5 (2013), 547-566.

[31] Sashi, C.M. Customer engagement, buyer-seller relationships, and social media. Management Decision 50, 2 (2012), 253-272.

[32] Trusov, M., Bodapati, A.V., and Bucklin, R.E. Determining Influential Users in Internet Social Networks. Journal of Marketing Research (JMR) 47, 4 (2010), 643-658.

[33] Xia, L. Effects of Companies’ Responses to Consumer Criticism in Social Media. International Journal of Electronic Commerce 17, 4 (2013), 73-100.

[34] Yu, Y., Duan, W., and Cao, Q. The impact of social and conventional media on firm equity value: A sentiment analysis approach. 1. Social Media Research and Applications 2. Theory and Applications of Social Networks 55, 4 (2013), 919-926.

[35] Zhao, D. and Rosson, M.B. How and Why People Twitter: The Role that Micro-blogging Plays in Informal Communication at Work. GROUP'04, (2009).

[36] Zhao, J., Wu, J., and Xu, K. Weak ties: Subtle role of information diffusion in online social networks. Phys. Rev. E 82, 1 (2010), 016105.
3. Moderate
4. High
5. Very High or Always

\section{Appendix B - Examples of shopping preferences questions}

Q1. What are your frequent online shopping websites? (Check all that apply)
- Amazon
○ Best Buy
- Walmart
○ Target 